\title{
Effects of fasting and refeeding on expression of MAFbx and MuRF1 in chick skeletal muscle
}

\author{
LI QingHe ${ }^{1}$, LI JinXiu ${ }^{1}$, LAN He ${ }^{1}$, WANG Nan ${ }^{2}$, HU XiaoXiang ${ }^{1}, \mathrm{CHEN} \mathrm{Li}^{3} \& \mathrm{LI} \mathrm{Ning}^{1^{*}}$ \\ ${ }^{1}$ State Key Laboratory for Agrobiotechnology, China Agricultural University, Beijing 100193, China; \\ ${ }^{2}$ College of Veterinary Medicine, China Agricultural University, Beijing 100193, China; \\ ${ }^{3}$ Institute of Animal Sciences and Veterinary Medicine, Zhejiang Academy of Agricultural Sciences, Hangzhou 310021, China
}

Received March 31, 2011; accepted August 1, 2011

\begin{abstract}
The present study investigated the effects of fasting and refeeding on the expression of proteasome-related genes and their downstream targets in the skeletal muscles of chicks. Seven-day-old chicks were fasted for 24 or $48 \mathrm{~h}$ and then refed for $4 \mathrm{~h}$. The expression levels of MAFbx and MuRF1, which function as E3 ligases in the ubiquitin-proteasome system, were investigated at the mRNA and protein levels. MAFbx and MuRF1 expression were increased by fasting and these increases were downregulated by refeeding. The expression of the target proteins of these E3 ligases, MyoD and M-CK, was also analyzed. The levels of these proteins were downregulated by fasting, and these decreases were rescued by refeeding. The results of this study indicate that fasting stimulates MAFbx and MuRF1 expression in chicks, possibly leading to increased degradation of their corresponding target proteins.
\end{abstract}

fasting, gene expression, MAFbx, MuRF1, MyoD, M-CK

Citation: Li Q H, Li J X, Lan H, et al. Effects of fasting and refeeding on expression of MAFbx and MuRF1 in chick skeletal muscle. Sci China Life Sci, 2011, 54: 904-907, doi: 10.1007/s11427-011-4226-2

MAFbx (muscle atrophy F-box) and MuRF1 (muscle RING finger 1) genes encode E3 ubiquitin ligases, which function in ubiquitin-mediated protein degradation in proteasomes. $M A F b x$, also known as atrogin1 and Fbxo32, contains an F-box domain that is characteristic of SCF-type E3 ubiquitin ligases $[1,2]$. MuRF1 is a muscle-specific RING-finger protein that binds the Cys-His zinc-binding motif [3]. $M A F b x$ and $M u R F 1$ are specifically expressed in cardiac and skeletal muscles and have vital roles in protein degradation during muscle wasting [4]. Numerous studies have demonstrated that the expression of these two genes is upregulated during processes that involve muscle wasting, including muscle atrophy, injury, burns, aging, chronic illness, and starvation $[1,5,6]$.

Proteins are mainly degraded via two systems in vivo, the

*Corresponding author (email: ninglbau@ public3.bta.net.cn) lysosome and the ubiquitin-proteasome systems, which are responsible for the degradation of extracellular and intracellular proteins, respectively [7]. Extracellular and cell membrane proteins are degraded by the lysosome proteolytic system, which includes several acid proteases and hydrolases [7]. In the ubiquitin-proteasome system, intracellular proteins are first marked by the covalent attachment of the 76-amino-acid ubiquitin protein via an isopeptide bond between the $\varepsilon$-amino group of a lysine of the substrate protein and the carboxyl terminus of ubiquitin, catalyzed by E3 ubiquitin ligases. More ubiquitin molecules attach to form a polyubiquitin chain on the targeted protein, which is subsequently recognized by the $26 \mathrm{~S}$ proteasome and degraded into small peptides [6]. MAFbx and MuRF1 are involved in the degradation of many proteins, especially myofibrillar proteins [8].

Skeletal muscle is the primary protein reservoir in 
mammals. Skeletal muscle protein shows high plasticity and can be mobilized into free amino acids for hepatic gluconeogenesis and energy production during starvation and pathological conditions [5]. Fasting increases the rate of protein degradation in skeletal muscle, while feeding conversely has a suppressive effect on proteolysis [9]. Evidence suggests that the transcription of $M A F b x$ is upregulated by fasting and that refeeding can rescue its overexpression [9]. However, the effects of fasting on the expression of $M u R F 1$ and the level of MAFbx protein remain to be elucidated.

As a muscle-specific transcription factor, MyoD is involved in cell cycle withdrawal and activation of muscle-specific genes that have important roles in skeletal muscle differentiation [10]. Muscle creatine kinase (M-CK) is a crucial enzyme in energy metabolism and catalyzes the reversible conversion of MgATP and creatine to MgADP and phosphocreatine [11]. Previous studies have demonstrated that MAFbx and MuRF1 degrade MyoD and M-CK, respectively, in skeletal muscle [10,12].

We therefore investigated the specific expression of MAFbx and MuRF1 and their downstream targets MyoD and $\mathrm{M}-\mathrm{CK}$ in chicken skeletal muscle during fasting and refeeding.

\section{Materials and methods}

\subsection{Animals}

One-day-old female broiler chicks were supplied by a local commercial hatchery (Huadu Chicks Company, Beijing). The chicks were fed with a semi-purified corn soybean meal diet and water for $7 \mathrm{~d}$. On day 7, chicks with similar body weights (about $70 \mathrm{~g}$ ) were selected and divided into four groups, with three chicks in each group: a normal feeding group (NF), a group deprived of food for $24 \mathrm{~h}$ (24F), a group deprived of food for $48 \mathrm{~h}(48 \mathrm{~F})$, and a group refed after $48 \mathrm{~h}$ of food deprivation (RF). Skeletal muscle tissues from each animal were flash frozen in liquid nitrogen and then stored at $-80^{\circ} \mathrm{C}$.

\subsection{RNA isolation}

Each tissue sample was ground in liquid nitrogen and the total RNA was extracted with TRIZOL ${ }^{\circledR}$ Reagent (Invitrogen). Aliquots of $30 \mu \mathrm{g}$ total RNA were digested with RNase-Free DNase I (NEB) for $15 \mathrm{~min}$ at $37^{\circ} \mathrm{C}$, followed by phenol-chloroform extraction and ethanol precipitation to remove DNA contamination. The concentration and quality of RNA were assessed using a Nanodrop 2000 spectrophotometer (Thermo Scientific) and by formaldehyde-agarose gel electrophoresis.

\subsection{Real-time polymerase chain reaction (PCR)}

First-strand cDNA was reverse transcribed using Moloney murine leukemia virus reverse transcriptase (Promega) and oligo (dT) primer (Promega). About $1.5 \mu \mathrm{g}$ of total RNA was used for each sample. Real-time PCR primers were designed using Oligo 6.0 software. The following primers were used in the experiments: MAFbx, forward: AGGCCGCAGTGTGTTGTTCT, and reverse: GTGTGAATGGCTGGTTGCAT; MuRF1, forward: GCCAAGCAGCTCATTAAAACG, and reverse: CATGTTCTCATAGCCTTGCTCAAT; M-CK, forward: CGGAGCACCTGGGTTACATC, and reverse: GGG GTGCTGGCTGAGTTTG; MyoD, forward: CAACAGCAGTGGTGT GACAGAT, and reverse: CAAAGCAACTCTTATTTACAATTATACA; GAPDH, forward: CGATCTGAACTACATGGTTTACATGTT, and reverse: CCCGTTCTCAGCCTTGACA. The expression of mRNA was measured by real-time PCR using an ABI 7900 HT instrument with the SYBR ${ }^{\circledR}$ Green system (Applied Biosystems). For PCR amplification, cDNA was predenatured at $95^{\circ} \mathrm{C}$ for $10 \mathrm{~min}$, followed by 40 cycles of $95^{\circ} \mathrm{C}$ for $30 \mathrm{~s}$, and $60^{\circ} \mathrm{C}$ for $1 \mathrm{~min}$. Expression of the housekeeping gene glyceraldehyde 3-phosphate dehydrogenase (GAPDH) was used as a reference to normalize the input cDNA. The relative expression values were analyzed using the comparative $\mathrm{Ct}$ method.

\subsection{Western blotting}

Animal tissues were first sheared using a Tissue Lyzer (Qiagen) in $1 \mathrm{~mL}$ lysis buffer $\left(150 \mathrm{mmol} \mathrm{L}{ }^{-1} \mathrm{NaCl}, 10 \mathrm{mmol}\right.$ $\mathrm{L}^{-1}$ Tris (pH 7.4), 5 mmol L ${ }^{-1}$ EDTA ( $\mathrm{pH} \mathrm{8.0),} 1 \%$ Triton $\mathrm{X}-100,1 \mathrm{mmol} \mathrm{L}^{-1}$ PMSF, $20 \mathrm{mg} \mathrm{mL}^{-1}$ aprotinin, $50 \mathrm{mg}$ $\mathrm{mL}^{-1}$ leupeptin, $1 \mathrm{mmol} \mathrm{L}^{-1}$ benzamidine, $1 \mathrm{mg} \mathrm{mL}^{-1}$ pepstatin). The lysates were incubated at room temperature for $30 \mathrm{~min}$ and then centrifuged for $5 \mathrm{~min}$ at $13000 \times \mathrm{g}$. The concentration of total protein in the supernatant was determined with the bicinchoninic acid assay (Beyotime Biotechnology). Proteins were normalized to $50 \mu \mathrm{g}$ in each lane, separated by $15 \%$ sodium dodecyl sulfate-polyacrylamide gel electrophoresis, and then transferred onto pure nitrocellulose blotting membranes (Millipore). After blocking with TBST (100 mmol L ${ }^{-1}$ Tris- $\mathrm{HCl}, \mathrm{pH} \mathrm{7.5,0.1 \%}$ Tween20, $0.9 \% \mathrm{NaCl})$ containing $5 \%(\mathrm{w} / \mathrm{v})$ skim milk for $1 \mathrm{~h}$, the membranes were incubated with primary antibodies against MAFbx (Abcam), MuRF1 (Santa Cruz) and $\alpha$-tubulin (Beyotime Biotechnology) respectively for $2 \mathrm{~h}$. They were then incubated with the corresponding secondary antibodies for $1 \mathrm{~h}$. After washing three times with TBST, detection was performed using a chemiluminescence ECL Westernblotting system (Roche), followed by exposure to autoradiographic film (Hyperfilm ECL, Amersham).

\subsection{Statistical analysis}

The results were expressed as mean \pm standard deviation (SD). The overall differences among groups were analyzed 
using analysis of variance (ANOVA). If the differences were significant, the differences between groups were estimated using the Student-Newman-Keuls multiple comparison post-hoc test. Results were considered to be significantly different if $P<0.05$.

\section{Results}

\subsection{Specific expression of MAFbx and MuRF1 in skele- tal muscle}

The protein expression levels of MAFbx and MuRF1 in cardiac muscle, liver, lung, skeletal muscle, large intestine, small intestine, glandular stomach, muscular stomach and kidney were investigated in chickens by Western blotting. Both MAFbx and MuRF1 were specifically expressed in skeletal muscle; no expression of either protein could be detected in cardiac muscle or skeletal muscle, when the same amount of protein was loaded onto the gel (Figure 1).

\subsection{Fasting-induced expression of MAFbx and MuRF1 RNAs and proteins}

The effects of fasting and refeeding on MAFbx and MuRF1 mRNA and protein expression in chick skeletal muscle were analyzed by quantitative-PCR and Western blotting, respectively. MAFbx and MuRFI mRNA levels were significantly increased by fasting. $M A F b x$ expression was increased about 5 -fold in the $24 \mathrm{~F}$ group $(P<0.01)$ and 6 -fold in the $48 \mathrm{~F}$ group $(P<0.01)$. When the chicks were refed for $4 \mathrm{~h}$ after $48 \mathrm{~h}$ of fasting, MAFbx induction was reduced dramatically to a lower level than that in the NF group (Figure 2A). MuRFI expression was increased about 7 -fold in the $24 \mathrm{~F}$ group $(P<0.01)$ and 34 -fold in the $48 \mathrm{~F}$ group $(P<0.01)$, and its expression was also reduced to a relatively low level by refeeding (Figure 2B). Thus refeeding significantly reduced both $M A F b x$ and $M u R F 1$ mRNAs to relatively low levels (Figure 2). Similarly, Western blotting indicated that MAFbx and MuRF1 protein levels in chick skeletal muscle were greatly increased by fasting, while refeeding inhibited the induction of MAFbx and MuRF1 (Figure 3A and B).

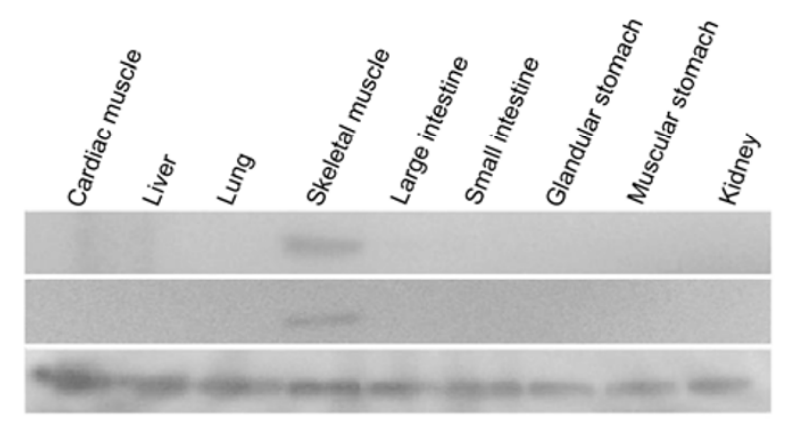

Figure 1 Expression of MAFbx and MuRF1 proteins in chick tissues detected by Western blotting.
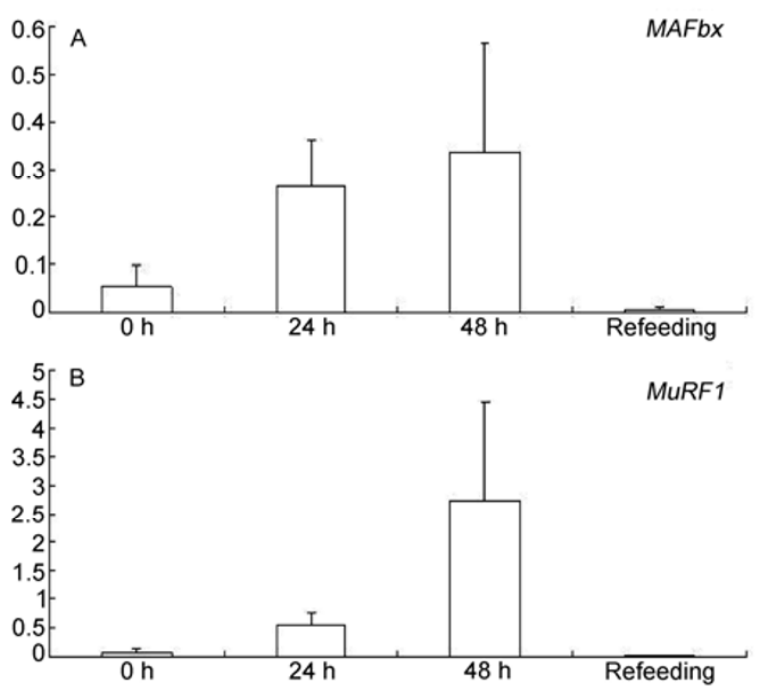

Figure 2 Induction of $M A F b x$ and $M u R F 1 \mathrm{mRNA}$ expression by fasting Each group included three replicates. A, MAFbx. B, MuRF1.

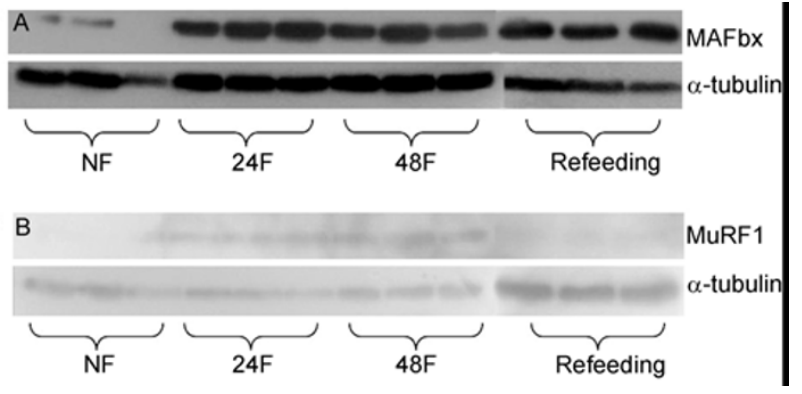

Figure 3 Induction of MAFbx and MuRF1 by fasting. A, MAFbx. B, MuRF1.

However, the rates of decrease in protein levels caused by refeeding were less obvious than those in RNA levels, possibly because of hysteresis of protein degradation.

\subsection{Downregulation of MyoD and M-CK in fasted skeletal muscle tissue}

Evidence suggests that MyoD and M-CK can be degraded by MAFbx and MuRF1, respectively, through the ubiquitin-proteasome system $[8,10,12]$. We therefore investigated changes in MyoD and M-CK expression in the skeletal muscles of chicks subjected to fasting and refeeding, using Western blotting. MyoD and M-CK expression levels showed opposite trends to those of MAFbx and MuRF1 (Figure 4). MyoD and M-CK protein levels decreased when chicks were fasted for $24 \mathrm{~h}$, and decreased further after fasting for $48 \mathrm{~h}$. These trends were reversed when the chicks were refed (Figure 4). However, neither fasting nor refeeding had any apparent effects on $M y o D$ and $M-C K$ mRNA levels (Figure 5A and B). 


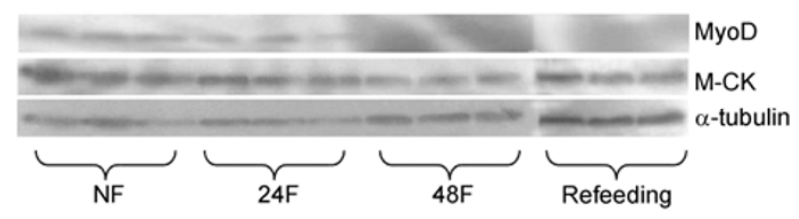

Figure 4 Downregulation of MyoD and M-CK by fasting.
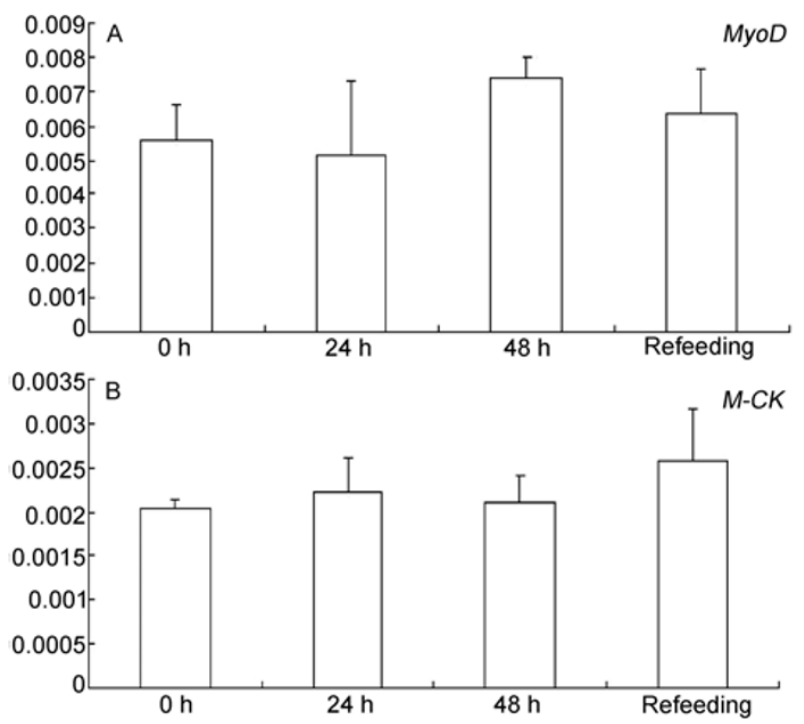

Figure 5 Effects of fasting on $M y o D$ and $M-C K$ mRNA levels. A, $M y o D$. $\mathrm{B}, M-C K$.

\section{Discussion}

This study investigated the short-term effects of fasting and refeeding on the metabolism of skeletal muscle protein in chicks. The E3 ligases MAFbx and MuRF1 were induced at the RNA and protein levels by fasting, while their corresponding target proteins MyoD and M-CK were downregulated at the protein, but not the mRNA level.

Muscle loss occurs as an important consequence of burns, chronic illness, injury, starvation and aging [1]. MAFbx and $M U R F 1$ were first demonstrated in muscle atrophy, and were found to be overexpressed in most states involving muscle loss, including paralysis, starvation, diabetes, sepsis, renal failure, and glucocorticoid excess [1]. Previous studies suggested that many genes related to the ubiquitinproteasome system were upregulated by starvation, including 14-kD E2, calpain large subunits, and $20 \mathrm{~S}$ proteasome subunits $[4,9]$. The current study demonstrated that, in accord with other components of the ubiquitin-proteasome system, the E3 ligases MAFbx and MuRF1 were significantly induced in chick skeletal muscle at both the RNA and protein levels following starvation for 12 and $24 \mathrm{~h}$. Refeeding normalized the expression of these two genes. These results demonstrate that the rates of protein synthesis and degradation in the skeletal muscle of growing animals are highly sensitive to food intake.
Evidence suggests that inhibition of MyoD degradation by MAFbx could prevent skeletal muscle atrophy in vivo [10]. We therefore investigated the effects of fasting and refeeding on the expression of the downstream proteins MyoD and M-CK, and demonstrated coordinated changes in the levels of these target proteins of MAFbx and MuRF1. MyoD is required to maintain the transcription of certain genes involved in cell cycle withdrawal and anti-apoptoticcell death in states of muscle loss, including the cyclin-dependent kinase inhibitor p21 and its downstream target, the retinoblastoma protein $[13,14]$. M-CK is an important skeletal muscle enzyme that has a crucial role in intracellular energy transport. We speculate that the fasting-induced downregulation of MyoD and M-CK proteins was caused by the upregulation of MAFbx and MuRF1. Although further studies are required, these results will help to further elucidate the role of the ubiquitin-proteasome system in the loss of skeletal muscle protein.

1 Bodine S C, Latres E, Baumhueter S, et al. Identification of ubiquitin ligases required for skeletal muscle atrophy. Science, 2001, 294: 1704-1708

2 Ilyin G P, Rialland M, Pigeon C, et al. cDNA cloning and expression analysis of new members of the mammalian F-box protein family. Genomics, 2000, 67: 40-47

3 Spencer J A, Eliazer S, Ilaria R L Jr, et al. Regulation of microtubule dynamics and myogenic differentiation by MURF, a striated muscle RING-finger protein. J Cell Biol, 2000, 150: 771-784

4 Kee A J, Combaret L, Tilignac T, et al. Ubiquitin-proteasomedependent muscle proteolysis responds slowly to insulin release and refeeding in starved rats. J Physiol, 2003, 546: 765-776

5 Gomes $\mathrm{M}$ D, Lecker $\mathrm{S} \mathrm{H}$, Jagoe $\mathrm{R} \mathrm{T}$, et al. Atrogin-1, a muscle-specific F-box protein highly expressed during muscle atrophy. Proc Natl Acad Sci USA, 2001, 98: 14440-14445

6 Zhao W, Wu Y, Zhao J, et al. Structure and function of the upstream promotor of the human Mafbx gene: the proximal upstream promotor modulates tissue-specificity. J Cell Biochem, 2005, 96: 209-219

7 Jackman R W, Kandarian S C. The molecular basis of skeletal muscle atrophy. Am J Physiol Cell Physiol, 2004, 287: C834-843

8 Witt S H, Granzier H, Witt C C, et al. MURF-1 and MURF-2 target a specific subset of myofibrillar proteins redundantly: towards understanding MURF-dependent muscle ubiquitination. J Mol Biol, 2005, 350: 713-722

9 Nakashima K, Yakabe Y, Yamazaki M, et al. Effects of fasting and refeeding on expression of atrogin-1 and Akt/FOXO signaling pathway in skeletal muscle of chicks. Biosci Biotechnol Biochem, 2006, 70: 2775-2778

10 Lagirand-Cantaloube J, Cornille K, Csibi A, et al. Inhibition of atrogin-1/MAFbx mediated MyoD proteolysis prevents skeletal muscle atrophy in vivo. PLoS ONE, 2009, 4: e4973

11 Zhao T J, Yan Y B, Liu Y, et al. The generation of the oxidized form of creatine kinase is a negative regulation on muscle creatine kinase. J Biol Chem, 2007, 282: 12022-12029

12 Koyama S, Hata S, Witt C C, et al. Muscle RING-finger protein-1 (MuRF1) as a connector of muscle energy metabolism and protein synthesis. J Mol Biol, 2008, 376: 1224-1236

13 Ishido M, Kami K, Masuhara M. Localization of MyoD, myogenin and cell cycle regulatory factors in hypertrophying rat skeletal muscles. Acta Physiol Scand, 2004, 180: 281-289

14 Ishido M, Kami K, Masuhara M. In vivo expression patterns of MyoD, p21, and $\mathrm{Rb}$ proteins in myonuclei and satellite cells of denervated rat skeletal muscle. Am J Physiol Cell Physiol, 2004, 287: C484-493

Open Access This article is distributed under the terms of the Creative Commons Attribution License which permits any use, distribution, and reproduction in any medium, provided the original author(s) and source are credited. 